\title{
Association between HLA genes and American cutaneous leishmaniasis in endemic regions of Southern Brazil
}

\author{
Rejane C Ribas-Silva ${ }^{1}$, Adriana D Ribas ${ }^{1}$, Maria CG dos Santos ${ }^{1}$, Waldir V da Silva $\mathrm{Jr}^{2}$, Maria VC Lonardoni ${ }^{3}$, \\ Sueli D Borelli $i^{*}$ and Thaís GV Silveira ${ }^{3}$
}

\begin{abstract}
Background: The present study sought to investigate the association between HLA-A, HLA-B and HLA-DRB1 genes and susceptibility or resistance to the different clinical manifestations of American cutaneous leishmaniasis (ACL) in southern Brazil.

Methods: The sample consisted of 169 patients with a diagnosis of ACL and 270 healthy subjects for comparison. HLA-A, HLA-B and HLA-DRB1 were typed by PCR-SSO reverse dot blot.

Results: Results showed a trend towards susceptibility to cutaneous lesions for alleles HLA-DRB1*13 ( $P=0.0228$; $\left.P_{C}=0.3420 ; O R=1.66 ; 95 \% C l=1.08-2.56\right), H L A-B * 35(P=0.0218 ; P C=0.6758 ; O R=1.67 ; 95 \% C l=1.08-2.29)$ and $\mathrm{HLA}-B^{*} 44$ $\left(P=0.0290 ; P_{C}=0.8990 ; O R=1.67 ; 95 \% C l=1.05-2.64\right)$. Subjects with allele HLA-B*27 $(P=0.0180 ; P C=0.5580 ; O R=7.111$; $95 \% \mathrm{Cl}=1.7850-28.3286)$ tended towards susceptibility to mucocutaneous lesions, those with HLA-B*49 $(P=0.0101$; $\left.P_{C}=0.3131 ; O R=6.4000 ; 95 \% C l=1.8472-22.1743\right)$ to recurrent $A C L$, and $H L A-B * 52(P=0.0044 ; P C=0.1360 ; O R=12.61$; $95 \% \mathrm{Cl}=3.08-51.66)$, to re-infection. Presence of $\mathrm{HLA}^{*} \mathrm{~B}^{*} 45(P=0.0107 ; P C=0.3317)$ tended to provide protection against the cutaneous form of $A C L$. The most frequent haplotypes that may be associated with susceptibility to $A C L$ were $A^{*} 02 B^{*} 44 D R B^{*} 07(P=0.0236)$ and $A^{*} 24 B^{*} 35 D^{2} B^{*} 01 \quad(P=0.0236)$.

Conclusion: Some Class I and Class II HLA genes appear to contribute towards susceptibility to and protection against different clinical manifestations of ACL. Other genetic marker studies may contribute toward future prophylactic and therapeutic interventions in ACL.
\end{abstract}

Keywords: Genetic susceptibility, HLA, Leishmaniasis, Leishmania brasiliensis

\section{Background}

American cutaneous leishmaniasis (ACL) is a noncontagious infectious disease, caused by several species of protozoa in the genus Leishmania and transmitted by sandfly bites, which causes skin and mucosal lesions [1-3].

In Brazil, ACL has been notified to the health authorities since the 1980s. Between 1990 and 2008, some 527,976 cases were notified, 12,115 of which in the southern region of Brazil, with 11,557 cases (95.4\%) from the state of Paraná alone [4]. The northern and western

\footnotetext{
* Correspondence: sdborelli@uem.br

${ }^{4}$ Immunogenetics Laboratory, Department of Basic Health Sciences, Universidade Estadual de Maringá (UEM), Av. Colombo, 5.790 - Jd. Universitário, Maringá, Paraná 87020-900, Brazil

Full list of author information is available at the end of the article
}

regions of the Southern state of Paraná stand out due to their high concentration of ACL cases, and are thus considered areas of epidemiological importance [5]. In these regions, the predominant species $(98.7 \%)$ is Leishmania (Viannia) braziliensis [6,7].

Infection by $L$. braziliensis may develop into the localized clinical cutaneous and/or mucosal forms of ACL. Cure of $\mathrm{ACL}$ is determined clinically, and is defined by regression of lesions within three months of concluding therapy. Periodical follow-up for 12 months is recommended. However, relapse of the disease may occur during the first year after cure. Relapse consists of the appearance of the cutaneous lesion at its former site or recurrence of mucosal lesions due to potential hematogenous spread of the infection [3]. It is estimated that relapse of cutaneous lesions occurs in
C Biomed Central 
approximately $10 \%$ of infected patients, whereas mucocutaneous lesion relapse, which constitutes the most severe complication of ACL, occurs in $4 \%$ of cases [8-11]. A distinct manifestation is recurrent ACL, which, unlike relapse, consists of the onset of cutaneous lesions in places other than those of the previous infection, possibly indicating reinfection [10].

The progression of ACL varies according to environmental factors, vector characteristics, parasite genetics and host immunological factors. Furthermore, patients infected with the same Leishmania species may develop different clinical forms of ACL even if they have the same nutritional conditions and are exposed to the same environmental factors [12-15]. In certain studies, HLA alleles have been associated with disease progression in cutaneous and mucocutaneous leishmaniasis [16-19]. In this context, the conduction of studies related to host genetic markers after exposure to the parasite is highly relevant.

Due to the dearth of data on genetic polymorphism of the HLA system and its association with protection against and/or susceptibility to development of ACL in the Brazilian population, the present study investigated possible involvements of Class I (HLA-A, HLA-B) and Class II (HLA-DRB1) HLA genes in the different clinical manifestations of ACL in populations of endemic regions in Southern Brazil where L. braziliensis is the predominant parasite.

\section{Methods}

\section{Patients and controls}

A retrospective study was carried out on the epidemiological records of patients from the $13^{\text {th }}$ and $15^{\text {th }}$ Health Sections of the state of Paraná diagnosed with ACL. The diagnosis was made at the Leishmaniases Laboratory of the State University of Maringá. Patients with clinical manifestations of the disease and with positive parasite detection and/or Montenegro skin test who received a medical prescription for treatment two years ago were selected. This period was defined because, according to WHO, more than $90 \%$ of recurrent cases occur up to one year after treatment.

Patient age at data collection ranged between 17 and 83 years (mean, $47.38 \pm 14.79$ ); 139 (82.3\%) patients were males and $30(17.8 \%)$ were females. According to ethnicity (skin color using Brazilian census categories), patient groups consisted of 127 (75.2\%) caucasian; 36 (21.3\%) amerindians; 4 (2.4\%) african and 2 (1.2\%) asian.

The control group comprised 260 healthy subjects, chosen according to age, gender, ethnicity, occupation and other demographic parameters, who had visited areas where ACL is endemic and had no clinical manifestations of the disease. Age ranged between 18 and 73 years (mean, $30.31 \pm 10.92)$; 141 controls $(54.2 \%)$ were males and 119 (45.8\%) were females. The ethnic distribution was 208 (80.0\%) white, 37 (14.2\%) brown, 8 (3.1\%) black and 7 (2.7\%) yellow.

Research subjects were interviewed and information was recorded in social-epidemiological files which included notes on clinical manifestations occurring after treatment, such as development of the mucosal form or even relapse. All subjects were informed of the investigation, and those who agreed to allow use of their data provided written informed consent. The study was approved by the Permanent Human Subject Research Ethics Committee of the State University of Maringá, with judgment number 153/2009.

\section{Group composition}

The study population comprised 169 subjects with a positive diagnosis of ACL who had received therapy and lived in Southern Brazil. The control group consisted of 260 subjects with no clinical manifestations of ACL who lived in the same area from which patients were recruited and who also frequented areas posing a high risk of infection (shrubland with nearby streams). Subjects belonging to both groups were unrelated.

According to clinical and laboratory data, patients were allocated into 4 groups and 6 subgroups (Figure 1).

Group 1 comprised subjects who had a cutaneous form of the disease and no evidence of any relapse. This group was subdivided into Group 1A, composed of 118 individuals who had a positive parasitological diagnosis, and Group 1B, composed of 18 individuals who had only a positive Montenegro test.

Group 2 consisted of subjects with clinical manifestations of mucosal disease. This group was subdivided into Group 2A, composed of 11 individuals with a positive laboratory diagnosis (parasite survey and/or Montenegro skin test) of a past cutaneous lesion with subsequent development of a mucosal lesion, and Group 2B, comprising 4 individuals who had a mucosal lesion but no history of previous cutaneous lesions.

Group 3 comprised individuals who had the cutaneous form of the disease and, after treatment, developed cutaneous lesions again. This group was subdivided into Group 3A, composed of 11 individuals who had a positive laboratory diagnosis (parasitological and/or Montenegro skin test) and lesion relapse at the original site, and Group $3 \mathrm{~B}$, composed of 7 individuals who had a positive laboratory diagnosis (parasitological and/or Montenegro skin test) and lesion relapse at a different site.

Group 4 comprised all subjects with a history of recurrent disease-Groups 2A and 3A. Recurrent ACL was defined as the onset of a new lesion after at least 1 year of treatment, whether at the same site of the previous lesion (Group 3A) or, subsequently, in the mucosa (Group 2A).

The small number of individuals in certain groups and subgroups of ACL was a limiting factor in this study, 


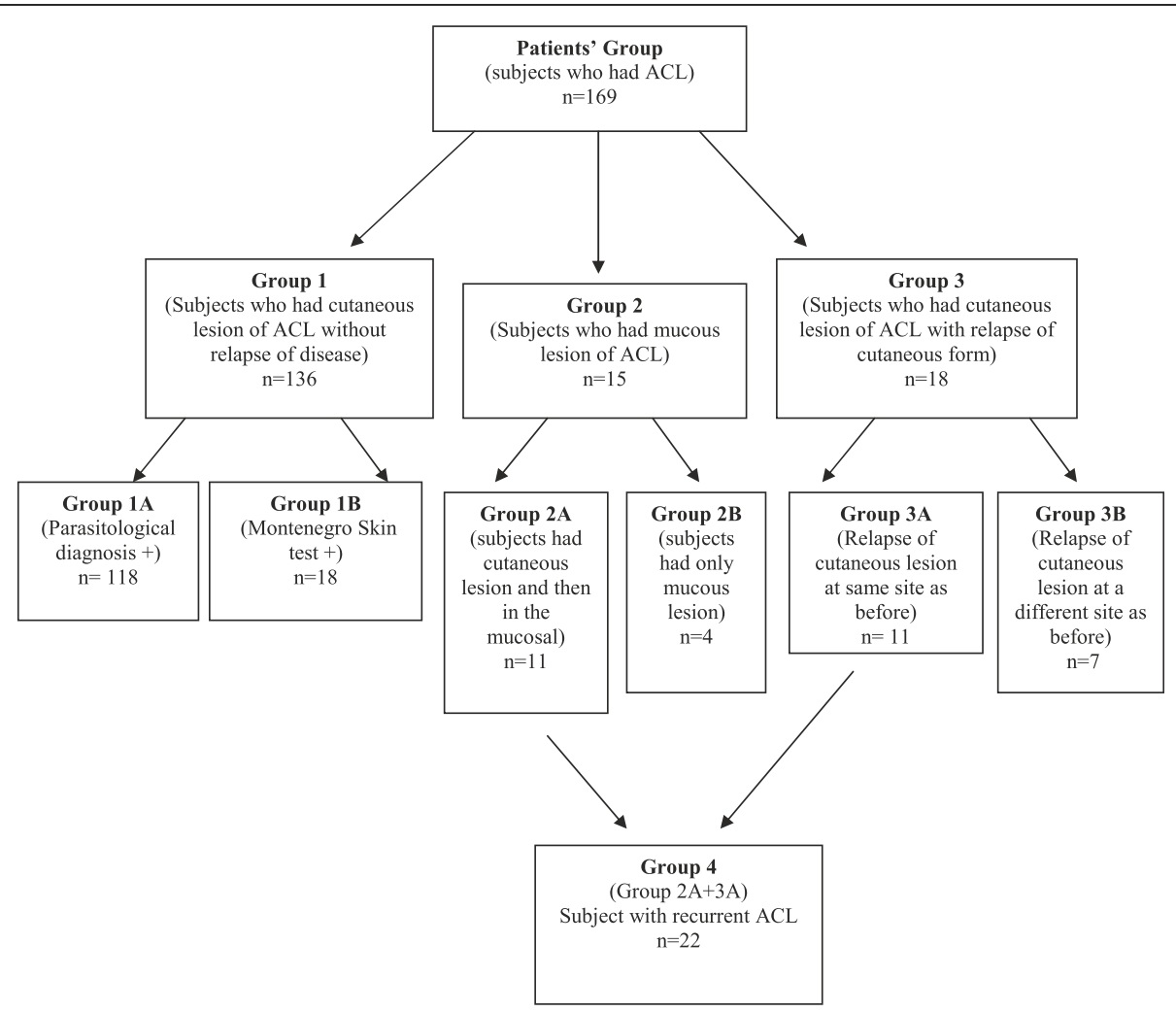

Figure 1 Composition of groups and subgroups comprising ACL group ( $n=169)$.

justified by the frequency of some clinical manifestations. Recurrence of ACL occurs in approximately $10 \%$ of infected patients, and mucocutaneous lesion relapse occurs in $4 \%$ of infected patients [8-11]. Thus, this study demonstrates the preliminary results of these groups.

\section{Determination of HLA alleles}

Peripheral blood samples $(10 \mathrm{~mL})$ were collected from patients and controls into EDTA anticoagulant tubes and centrifuged at $960 \mathrm{~g}$ for 10 minutes. Nucleated cells were separated and frozen at $-80^{\circ} \mathrm{C}$ until use. Genomic DNA was extracted from $100 \mu \mathrm{L}$ aliquots of frozen cells with the EZ-DNA extraction kit (Biological Industries ${ }^{\circledR}$, Kibbutz Beit Haemek, Israel), according to manufacturer instructions.

Patients' and controls' HLA Class I (A, B) and Class II (DRB1) genotypes were determined by the reverse dot blot PCR-SSO method, using the commercially available RELI ${ }^{\mathrm{mm}}$ SSO kit (DYNAL BIOTECH A.S.A, Oslo, Norway). Genotyping was performed at the Immunogenetic Laboratory of the State University of Maringá.

\section{Statistical analysis}

A data bank of HLA-A, -B and -DRB1 processing results was created in Microsoft Excel 2007. Data were expressed as allele frequency $(F)$, calculated as the ratio of the number of times different alleles appeared in the sample to the total number of alleles. The $P$-value was calculated using a two-tailed Fisher's exact test at a $5 \%$ significance level. Significant $P$-values were corrected with the Bonferroni method $(P \mathrm{c})$ by multiplying $P$ by the number of alleles detected for each locus. Again, PC values $<0.05$ were considered significant. Odds ratios (OR) at a 95\% confidence interval (CI) were calculated for results with $P<0.05$. Whereas descriptive statistical analyses for sex and ethnicity variables were determined by Fisher's exact test, the age variable was calculated with the $t$-test for independent samples, also with a significance level of $5 \%$. Statistical analyses were performed in the $R$ and Statistica 7.0 software packages. Arlequin 2000 http://cmpg.unibe.ch/software/arlequin3/ was employed to confirm the distribution of allele frequency and $P>0.05$ rates were in Hardy-Weinberg equilibrium (HW). Haplotype frequencies were estimated using the Expectation Maximization (EM) algorithm included in the Arlequin software.

\section{Results}

The present study investigated a potential association between ACL and Class I and Class II HLA alleles in a Southern Brazilian population by comparing the frequency of HLA-A, HLA-B and HLA-DRB1 alleles in 
subjects affected by the disease and those of healthy controls.

The distribution of allele proportions reported is in HW equilibrium both in the patient group and in the control one.

\section{ACL overall versus control group}

Table 1 shows the HLA-A, HLA-B and HLA-DRB1 allele frequencies of the group. Overall, there were differences between ACL patients and the control group for allele HLA-B* 45 , which was more frequent in the control group [0\% vs. $2.31 \%(P=0.0046$ and $P c=0.1426)]$, with a trend towards resistance to ACL development. The presence of HLA-DRB1*13 in the ACL and control groups [15.1\% vs. $10.38 \%(P=0.0431 ; P c=0.6465 ; \mathrm{OR}=1.5335 ; 95 \% \mathrm{CI}=$ 1.0177 - 2.3107)] suggested a trend towards ACL susceptibility.

Table 1 Frequency of HLA-A, -B and -DRB1 alleles in the ACL group versus the control group

\begin{tabular}{|c|c|c|c|c|c|c|c|c|c|c|c|c|c|c|c|c|c|}
\hline \multirow[b]{2}{*}{ Allele } & \multicolumn{2}{|c|}{$\mathrm{ACL}(\mathrm{n}=169)$} & \multicolumn{2}{|c|}{$\begin{array}{l}\text { Controls } \\
(n=260)\end{array}$} & \multirow[b]{2}{*}{$P$-value } & \multirow[b]{2}{*}{ Pc-value } & \multirow[b]{2}{*}{ OR } & \multirow[b]{2}{*}{$95 \% \mathrm{Cl}$} & \multirow[b]{2}{*}{ Allele } & \multicolumn{2}{|c|}{$\mathrm{ACL}(\mathrm{n}=169)$} & \multicolumn{2}{|c|}{$\begin{array}{l}\text { Controls } \\
(\mathrm{n}=260)\end{array}$} & \multirow[b]{2}{*}{$P$-value } & \multirow[b]{2}{*}{ Pc-value } & \multirow[b]{2}{*}{ OR } & \multirow[b]{2}{*}{$95 \% \mathrm{Cl}$} \\
\hline & $n$ & $F \%$ & $n$ & $F \%$ & & & & & & $\mathrm{n}$ & $F \%$ & $\mathrm{n}$ & $F \%$ & & & & \\
\hline HLA - $A^{*}$ & & & & & & & & & HLA-B* & & & & & & & & \\
\hline 01 & 37 & 10.9 & 47 & 9.0 & ns & - & & & 07 & 20 & 5.9 & 38 & 7.3 & ns & - & & \\
\hline 02 & 95 & 28.1 & 127 & 24.4 & ns & - & & & 08 & 16 & 4.7 & 34 & 6.5 & ns & - & & \\
\hline 03 & 30 & 8.8 & 56 & 10.8 & ns & - & & & 13 & 1 & 0.3 & 9 & 1.7 & ns & - & & \\
\hline 11 & 23 & 6.8 & 26 & 5.0 & ns & - & & & 14 & 15 & 4.4 & 22 & 4.2 & ns & - & & \\
\hline 23 & 18 & 5.3 & 22 & 4.2 & ns & - & & & 15 & 26 & 7.7 & 48 & 9.2 & ns & - & & \\
\hline 24 & 37 & 10.9 & 62 & 11.9 & ns & - & & & 18 & 17 & 5.0 & 36 & 6.9 & ns & - & & \\
\hline 25 & 4 & 1.2 & 13 & 2.5 & ns & - & & & 27 & 7 & 2.1 & 8 & 1.5 & ns & - & & \\
\hline 26 & 11 & 3.2 & 18 & 3.5 & ns & - & & & 35 & 58 & 17.2 & 68 & 13.1 & ns & - & & \\
\hline 29 & 9 & 2.6 & 28 & 5.4 & ns & - & & & 37 & 1 & 0.3 & 5 & 1.0 & ns & - & & \\
\hline 30 & 11 & 3.2 & 25 & 4.8 & ns & - & & & 38 & 10 & 3.0 & 15 & 2.9 & ns & - & & \\
\hline 31 & 18 & 5.3 & 19 & 3.7 & ns & - & & & 39 & 13 & 3.9 & 18 & 3.5 & ns & - & & \\
\hline 32 & 8 & 2.3 & 16 & 3.1 & ns & - & & & 40 & 18 & 5.3 & 16 & 3.1 & ns & - & & \\
\hline 33 & 5 & 1.5 & 15 & 2.9 & ns & - & & & 41 & 2 & 0.6 & 6 & 1.2 & ns & - & & \\
\hline 34 & 3 & 0.9 & 5 & 1.0 & ns & - & & & 42 & 2 & 0.6 & 6 & 1.2 & ns & - & & \\
\hline 36 & 2 & 0.6 & 2 & 0.4 & ns & - & & & 44 & 43 & 12.7 & 46 & 8.9 & ns & - & & \\
\hline 39 & 1 & 0.3 & - & - & ns & - & & & 45 & 0 & - & 12 & 2.3 & 0.0046 & 0.1426 & - & - \\
\hline 66 & 3 & 0.9 & 1 & 0.2 & ns & - & & & 46 & 0 & - & 4 & 0.8 & ns & - & & \\
\hline 68 & 19 & 5.62 & 35 & 6.7 & ns & - & & & 47 & 0 & - & 1 & 0.2 & ns & - & & \\
\hline 74 & 4 & 1.9 & 3 & 0.6 & ns & - & & & 48 & 3 & 0.9 & 2 & 0.4 & ns & - & & \\
\hline HLA-DRB1* & & & & & & & & & 49 & 8 & 2.4 & 8 & 1.5 & ns & - & & \\
\hline 01 & 23 & 6.8 & 42 & 8.1 & ns & - & & & 50 & 8 & 2.4 & 14 & 2.7 & ns & - & & \\
\hline 03 & 32 & 9.5 & 61 & 11.7 & ns & - & & & 51 & 29 & 8.6 & 44 & 8.5 & ns & - & & \\
\hline 04 & 40 & 11.8 & 56 & 10.8 & ns & - & & & 52 & 10 & 3.0 & 11 & 2.1 & ns & - & & \\
\hline 07 & 39 & 11.5 & 67 & 12.9 & ns & - & & & 53 & 7 & 2.1 & 8 & 1.5 & ns & - & & \\
\hline 08 & 26 & 7.7 & 30 & 5.8 & ns & - & & & 54 & 1 & 0.3 & 3 & 0.6 & ns & - & & \\
\hline 09 & 4 & 1.2 & 4 & 0.8 & ns & - & & & 55 & 8 & 2.4 & 12 & 2.3 & ns & - & & \\
\hline 10 & 5 & 1.5 & 10 & 1.9 & ns & - & & & 56 & 2 & 0.6 & 1 & 0.2 & ns & - & & \\
\hline 11 & 45 & 13.3 & 81 & 15.6 & ns & - & & & 57 & 7 & 2.1 & 12 & 2.3 & ns & - & & \\
\hline 12 & 3 & 0.9 & 10 & 1.9 & ns & - & & & 58 & 6 & 1.8 & 10 & 1.9 & ns & - & & \\
\hline 13 & 51 & 15.1 & 54 & 10.4 & 0.0431 & 0.6465 & 1.53 & $1.01-2.31$ & 73 & 0 & - & 1 & 0.2 & ns & - & & \\
\hline 14 & 19 & 5.6 & 40 & 7.7 & ns & - & & & 81 & 0 & - & 2 & 3.9 & ns & - & & \\
\hline 15 & 34 & 10.1 & 44 & 8.8 & ns & - & & & & & & & & & & & \\
\hline 16 & 17 & 5.0 & 21 & 4.0 & ns & - & & & & & & & & & & & \\
\hline
\end{tabular}


Haplotype analysis revealed frequency of 772 haplotypes in the ACL group and 1118 in the control group. Only haplotypes whose frequencies appeared to differ between groups (17 haplotypes) were analyzed. There were statistical differences between patients with ACL and controls for the haplotypes $A^{*} 02 \quad B^{*} 44$ DRB1*07 (2.37\% vs. $0 \%[P=0.0236])$ and $A^{*} 24 B^{*} 35$ DRB1*01 (2.37\% vs. $0 \%[P=0.0236])$. Thus, these two haplotypes may be involved in susceptibility to ACL (Table 2).

\section{Group 1 versus control group}

Table 3 shows a comparison of HLA-A, HLA-B and HLA-DRB1 allele frequencies between Group 1 and the control group. Differences exist between Group 1 and the control group for HLA-B*35, which was more frequent in patients $\left[19.5 \%\right.$ vs. $13.1 \%\left(P=0.0218\right.$; $P_{C}=$ $0.6758 ; \mathrm{OR}=1.6086 ; 95 \% \mathrm{CI}=1.0851-2.3848)$, and HLA-B $* 4$ [ $13.9 \%$ vs. $8.8 \%(P=0.0290$; Pc $=0.8990 ;$ OR $=$ $1.6734 ; 95 \% \mathrm{CI}=1.0592-2.6436)$, which suggests susceptibility to the cutaneous form of ACL. HLA-B*45 was only present in controls $[0 \%$ vs. $2.31 \%(P=0.0107 ; P c=$ $0.3317)$ ], which suggests that it may confer resistance to the cutaneous form of ACL. Moreover, comparison of HLA-DRB1*13 frequency in group 1 versus controls [16.2\% vs. $10.4 \%(P=0.0228 ; P c=0.3420 ; \mathrm{OR}=1.6654$; $95 \% \mathrm{CI}=1.0851-2.5561)$ indicated a possible susceptibility to the cutaneous form of ACL.

\section{Subgroup $1 \mathrm{~A}$ versus control group}

Table 4 shows HLA-A, HLA-B and HLA-DRB1 allele frequencies in subgroup $1 \mathrm{~A}$ and the control group. When allele frequencies were compared between group $1 \mathrm{~A}$ and the control group, HLA-B* 44 [ $14.8 \%$ vs. $8.8 \%(P=0.0160$; $P c=0.4960 ; \mathrm{OR}=1.7943 ; 95 \% \mathrm{CI}=1.1219-2.8696)] \mathrm{sug}-$ gested a possible susceptibility to the cutaneous form of ACL. On the other hand, HLA-B*45 [0\% vs., 2.31\% ( $P=$ 0.0225; $P c=0.6975)$ suggested possible resistance to the cutaneous form of ACL. HLA-DRB1*13 [16.10\% vs. $10.38 \%(P=0.0152 ; P c=0.2280 ; \mathrm{OR}=1.6562 ; 95 \% \mathrm{CI}=$ $1.0591-2.5899)$ may also be involved in susceptibility to the cutaneous form of ACL.

Preliminary results from the other groups and subgroups versus the control group

Analysis of allele frequencies in subgroups 1B, 2A, 3A and group 3 versus the control group did not reveal any statistically significant differences in the HLA loci studied.

Comparison between group 2 and the control group showed that HLA-B*27 allele was more frequent in group 2 subjects $(10.0 \%$ vs. $1.5 \%[P=0.0180 ; P c=$ 0.5580 ; $\mathrm{OR}=7.1111 ; 95 \% \mathrm{CI}=1.7850-28.3286])$ indicating a possible susceptibility to mucocutaneous ACL. Furthermore, the HLA-B*27 allele was more frequent in patients of subgroup $2 \mathrm{~B}\left(25.0 \%\right.$ vs. $1.5 \%\left[P=0.0085 ; P_{C}=\right.$ $0.2635 ;$ OR $=21.3333 ;$ IC $95 \%=3.7218-122.2835]$ ), suggesting susceptibility to mucocutaneous ACL.

HLA-A*01 was more frequent in patients from subgroup $3 \mathrm{~B}(28.6 \%$ vs. $9 \%[P=0.0360 ; P c=0.6480 ; \mathrm{OR}=$ $4.0255 ; 95 \% \mathrm{CI}=1.2153-13.3341])$, as was HLA-B*52 (21.4\% vs. $2.1 \%[P=0.0044 ; P c=0.1364 ;$ OR $=12.6198$; $95 \%$ CI $=3.0827-51.6619]$ ), suggesting possible susceptibility to relapse of cutaneous ACL at a location remote from the site of previous infection.

HLA-B*49 was more frequent in group 4 patients than in controls $(9.1 \%$ vs. $1.5 \%[P=0.0101 ; P c=0.3131 ; \mathrm{OR}=$ $6.4000 ; 95 \% \mathrm{CI}=1.8472-22.1743]$ ), indicating susceptibility to recurrent ACL.

After correction of $P$-values, we could not detect any association between susceptibility to or protection against ACL within the allele frequencies of MHC class I (HLA-A and HLA-B) and class II (HLA-DRB1) genetic markers among the several the patient and control groups and subgroups analyzed in this study. Table 5 shows all alleles with trends toward increased susceptibility and/or resistance to ACL in these groups and subgroups.

\section{Discussion}

One of the first studies assessing the potential association between HLA and cutaneous leishmaniasis involving serological methods was undertaken in France, with L. guyanensis. The study revealed a low HLA-Cw7 frequency was associated with pathogenesis of cutaneous leishmaniasis [16]. A Mexican study involving $L$. mexicana was undertaken by the molecular biology method, with 65 cases of cutaneous leishmaniasis and 100 controls. Whereas the frequencies of two alleles were associated with the protection against cutaneous leishmaniasis, namely HLA-DR2 (OR $=0.15$; 95\%CI = $0.05-0.38 ; P=0.0000018)$ and HLA-DPB1*0401 (OR = $0.37 ; 95 \% \mathrm{CI}=0.21-0.67 ; P=0.0004)$, two others were

Table 2 Frequency of HLA-A/-B/-DRB1 haplotypes in the ACL group versus the control group

\begin{tabular}{|c|c|c|c|c|}
\hline \multirow[t]{2}{*}{ Haplotypes } & \multicolumn{4}{|c|}{ Frequency (\%) } \\
\hline & Controls $(n=260)$ & $A C L(n=169)$ & $P$-value & OR $(95 \% \mathrm{Cl})$ \\
\hline HLA-A*02-B*44-DRB1*07 & $0(0.00 \%)$ & $4(2.37 \%)$ & 0.0236 & - \\
\hline HLA-A*24-B*35-DRB1*01 & $0(0.00 \%)$ & $4(2.37 \%)$ & 0.0236 & - \\
\hline
\end{tabular}

$P$-value obtained by Fisher's exact test. Of all analyzed haplotypes, only those having $P$-values $<0.05$ were added to this table. 
Table 3 Frequency of HLA-A, -B and -DRB1 alleles in ACL Group 1 and the control group

\begin{tabular}{|c|c|c|c|c|c|c|c|c|c|c|c|c|c|c|c|c|c|}
\hline \multirow[b]{2}{*}{ Allele } & \multicolumn{2}{|c|}{$\begin{array}{c}\text { ACL (group 1) } \\
(n=136)\end{array}$} & \multicolumn{2}{|c|}{$\begin{array}{l}\text { Controls } \\
(n=260)\end{array}$} & \multirow[b]{2}{*}{$P$-value } & \multirow[b]{2}{*}{ Pc-value } & \multirow[b]{2}{*}{ OR } & \multirow[b]{2}{*}{$95 \% \mathrm{Cl}$} & \multirow[b]{2}{*}{ Allele } & \multicolumn{2}{|c|}{$\begin{array}{c}\text { ACL (group 1) } \\
(n=136)\end{array}$} & \multicolumn{2}{|c|}{$\begin{array}{l}\text { Controls } \\
(n=260)\end{array}$} & \multirow[b]{2}{*}{$P$-value } & \multirow[b]{2}{*}{ Pc-value } & \multirow[b]{2}{*}{ OR } & \multirow[b]{2}{*}{$95 \% \mathrm{Cl}$} \\
\hline & $\mathrm{n}$ & $F \%$ & $\mathrm{n}$ & $F \%$ & & & & & & $\mathrm{n}$ & $F \%$ & $n$ & F\% & & & & \\
\hline $\mathrm{HLA}-\mathrm{A}^{*}$ & & & & & & & & & HLA-B* & & & & & & & & \\
\hline 01 & 29 & 10.7 & 47 & 9.0 & ns & - & & & 07 & 15 & 5.5 & 38 & 7.3 & ns & - & & \\
\hline 02 & 73 & 26.8 & 127 & 24.4 & ns & - & & & 08 & 10 & 3.7 & 34 & 6.5 & ns & - & & \\
\hline 03 & 24 & 8.8 & 56 & 10.8 & ns & - & & & 13 & 1 & 0.4 & 9 & 1.7 & ns & - & & \\
\hline 11 & 18 & 6.6 & 26 & 5.0 & ns & - & & & 14 & 14 & 5.2 & 22 & 4.2 & ns & - & & \\
\hline 23 & 14 & 5.2 & 22 & 4.2 & ns & - & & & 15 & 23 & 8.5 & 48 & 9.2 & ns & - & & \\
\hline 24 & 28 & 10.3 & 62 & 11.9 & ns & - & & & 18 & 13 & 4.8 & 36 & 6.9 & ns & - & & \\
\hline 25 & 4 & 1.5 & 13 & 2.5 & ns & - & & & 27 & 3 & 1.1 & 8 & 1.5 & ns & - & & \\
\hline 26 & 8 & 2.9 & 18 & 3.5 & ns & - & & & 35 & 53 & 19.5 & 68 & 13.1 & 0.0218 & 0.6758 & 1.67 & $1.08-2.29$ \\
\hline 29 & 9 & 3.3 & 28 & 5.4 & ns & - & & & 37 & 1 & 0.4 & 5 & 1.0 & ns & - & & \\
\hline 30 & 9 & 3.3 & 25 & 4.8 & ns & - & & & 38 & 7 & 2.6 & 15 & 2.9 & ns & - & & \\
\hline 31 & 15 & 5.5 & 19 & 3.7 & ns & - & & & 39 & 10 & 3.7 & 18 & 3.5 & ns & - & & \\
\hline 32 & 7 & 2.6 & 16 & 3.1 & ns & - & & & 40 & 14 & 5.2 & 16 & 3.1 & ns & - & & \\
\hline 33 & 5 & 1.8 & 15 & 2.9 & ns & - & & & 41 & 2 & 0.7 & 6 & 1.2 & ns & - & & \\
\hline 34 & 3 & 1.1 & 5 & 1.0 & ns & - & & & 42 & 2 & 0.7 & 6 & 1.2 & ns & - & & \\
\hline 36 & 1 & 0.4 & 2 & 0.4 & ns & - & & & 44 & 38 & 14.0 & 46 & 8.9 & 0.0290 & 0.8990 & 1.67 & $1.05-2.64$ \\
\hline 39 & 1 & 0.4 & - & - & ns & - & & & 45 & 0 & - & 12 & 2.3 & 0.0107 & 0.3317 & - & - \\
\hline 66 & 2 & 0.7 & 1 & 0.2 & ns & - & & & 46 & 0 & - & 4 & 0.8 & ns & - & & \\
\hline 68 & 19 & 7.0 & 35 & 6.7 & ns & - & & & 47 & 0 & - & 1 & 0.2 & ns & - & & \\
\hline 74 & 3 & 1.1 & 3 & 0.6 & ns & - & & & 48 & 3 & 1.1 & 2 & 0.4 & ns & - & & \\
\hline HLA-DRB1* & & & & & & & & & 49 & 4 & 1.5 & 8 & 1.5 & ns & - & & \\
\hline 01 & 18 & 6.6 & 42 & 8.1 & ns & - & & & 50 & 6 & 2.2 & 14 & 2.7 & ns & - & & \\
\hline 03 & 22 & 8.1 & 61 & 11.7 & ns & - & & & 51 & 24 & 8.8 & 44 & 8.5 & ns & - & & \\
\hline 04 & 33 & 12.1 & 56 & 10.8 & ns & - & & & 52 & 6 & 2.2 & 11 & 2.1 & ns & - & & \\
\hline 07 & 33 & 12.1 & 67 & 12.9 & ns & - & & & 53 & 7 & 2.6 & 8 & 1.5 & ns & - & & \\
\hline 08 & 20 & 7.4 & 30 & 5.8 & ns & - & & & 54 & 0 & - & 3 & 0.6 & ns & - & & \\
\hline 09 & 4 & 1.5 & 4 & 0.8 & ns & - & & & 55 & 5 & 1.8 & 12 & 2.3 & ns & - & & \\
\hline 10 & 5 & 1.8 & 10 & 1.9 & ns & - & & & 56 & 1 & 0.3 & 1 & 0.2 & ns & - & & \\
\hline 11 & 33 & 12.1 & 81 & 15.6 & ns & - & & & 57 & 5 & 1.8 & 12 & 2.3 & ns & - & & \\
\hline 12 & 3 & 1.1 & 10 & 1.9 & ns & - & & & 58 & 5 & 1.8 & 10 & 1.9 & ns & - & & \\
\hline
\end{tabular}


Table 3 Frequency of HLA-A, -B and -DRB1 alleles in ACL Group 1 and the control group (Continued)

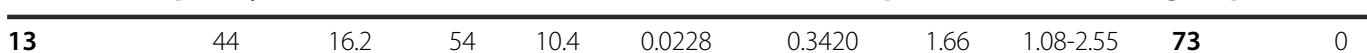

81

0.2

ns

$\begin{array}{lllllllllllll}14 & 14 & 5.2 & 40 & 7.7 & \text { ns } & - & \mathbf{8 1} & 0 & - & 2 & 3.9 & \text { ns }\end{array}$

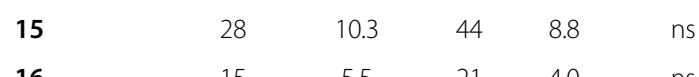

$\mathrm{n}=$ number of times the allele occurs; $\mathrm{F}=$ allele frequency; $P$-value $=$ calculated by Fisher's exact test; $\mathrm{Pc}$-value $=$ value of $P$ corrected by Bonferroni correction; $\mathrm{OR}=$ odds ratio; $95 \% \mathrm{Cl}=95 \%$ confidence interval; $\mathrm{ns}=$ not significant $(p>0.05)$. 
Table 4 Frequency of HLA-A, -B and -DRB1 alleles in ACL subgroup $1 A$ and control group

\begin{tabular}{|c|c|c|c|c|c|c|c|c|c|c|c|c|c|c|c|c|c|}
\hline \multirow[b]{2}{*}{ Allele } & \multicolumn{2}{|c|}{$\begin{array}{c}\mathrm{ACL}(\text { group } 1 \mathrm{~A}) \\
(\mathrm{n}=118)\end{array}$} & \multicolumn{2}{|c|}{$\begin{array}{l}\text { Controls } \\
(n=260)\end{array}$} & \multirow[b]{2}{*}{$P$-value } & \multirow[b]{2}{*}{ Pc-value } & \multirow[b]{2}{*}{ OR } & \multirow[b]{2}{*}{$95 \% \mathrm{Cl}$} & \multirow[b]{2}{*}{ Allele } & \multicolumn{2}{|c|}{$\begin{array}{c}\mathrm{ACL}(\text { group } 1 \mathrm{~A}) \\
(\mathrm{n}=118)\end{array}$} & \multicolumn{2}{|c|}{ Controls } & \multirow[b]{2}{*}{$P$-value } & \multirow[b]{2}{*}{ Pc-value } & \multirow[b]{2}{*}{ OR } & \multirow[b]{2}{*}{$95 \% \mathrm{Cl}$} \\
\hline & $n$ & $F \%$ & $n$ & $F \%$ & & & & & & $n$ & $F \%$ & $n$ & $F \%$ & & & & \\
\hline $\mathrm{HLA}-\mathrm{A}^{*}$ & & & & & & & & & HLA-B* & & & & & & & & \\
\hline 01 & 26 & 11.0 & 47 & 9.0 & ns & - & & & 07 & 14 & 5.9 & 38 & 7.3 & ns & - & & \\
\hline 02 & 64 & 27.1 & 127 & 24.4 & ns & - & & & 08 & 10 & 4.2 & 34 & 6.5 & ns & - & & \\
\hline 03 & 21 & 8.9 & 56 & 10.8 & ns & - & & & 14 & 10 & 4.2 & 22 & 4.2 & ns & - & & \\
\hline 11 & 16 & 6.8 & 26 & 5.0 & ns & - & & & 15 & 22 & 9.3 & 48 & 9.2 & ns & - & & \\
\hline 24 & 24 & 10.2 & 62 & 11.9 & ns & - & & & 18 & 10 & 4.2 & 36 & 6.9 & ns & - & & \\
\hline HLA-DRB1* & & & & & & & & & 27 & 3 & 1.3 & 8 & 1.5 & ns & - & & \\
\hline 01 & 16 & 6.8 & 42 & 8.1 & $\mathrm{~ns}$ & & & & 35 & 44 & 18.6 & 68 & 13.1 & ns & - & & \\
\hline 03 & 20 & 8.4 & 61 & 11.7 & ns & - & & & 39 & 8 & 3.4 & 18 & 3.5 & ns & - & & \\
\hline 04 & 27 & 11.4 & 56 & 10.8 & ns & - & & & 40 & 12 & 5.1 & 16 & 3.1 & ns & - & & \\
\hline 07 & 29 & 12.3 & 67 & 12.9 & ns & - & & & 44 & 35 & 14.8 & 46 & 8.9 & 0.0160 & 0.4969 & 1.79 & $1.12-2.87$ \\
\hline 08 & 17 & 7.2 & 30 & 5.8 & ns & - & & & 45 & 0 & - & 12 & 2.3 & 0.0231 & 0.0225 & - & - \\
\hline 11 & 31 & 13.1 & 81 & 15.6 & ns & - & & & 49 & 3 & 1.3 & 8 & 1.5 & ns & - & & \\
\hline 13 & 38 & 16.1 & 54 & 10.4 & 0.0152 & 0.2280 & 1.65 & $1.06-2.59$ & 51 & 20 & 8.5 & 44 & 8.5 & ns & - & & \\
\hline 14 & 11 & 4.7 & 40 & 7.7 & ns & & & & 52 & 6 & 2.5 & 11 & 2.1 & ns & - & & \\
\hline 15 & 24 & 10.2 & 44 & 8.8 & ns & - & & & & & & & & & & & \\
\hline
\end{tabular}

not significant $(p>0.05)$. This table is restricted to representative allele frequencies. 
Table 5 Contribution of Class I and II HLA alleles to development of ACL in a Southern Brazilian population

\begin{tabular}{|c|c|c|c|c|c|c|c|c|}
\hline \multirow{2}{*}{ Allele } & \multicolumn{2}{|c|}{$\mathrm{ACL}$} & \multicolumn{2}{|c|}{ Controls $(n=260)$} & \multirow[t]{2}{*}{$P$-value } & \multirow[t]{2}{*}{ Pc-value } & \multirow[t]{2}{*}{ OR } & \multirow[t]{2}{*}{$95 \% \mathrm{Cl}$} \\
\hline & $n$ & $F \%$ & $\mathrm{n}$ & $F \%$ & & & & \\
\hline \multicolumn{9}{|c|}{ ACL overall $(n=169)$} \\
\hline $\mathrm{HLA}-\mathrm{B}^{*} 45$ & 0 & - & 12 & 2.3 & 0.0046 & 0.1426 & - & - \\
\hline HLA-DRB $1 * 13$ & 51 & 15.1 & 54 & 10.4 & 0.0431 & 0.6465 & 1.53 & $1.01-2.31$ \\
\hline \multicolumn{9}{|c|}{$\mathrm{ACL}$ (group $1 ; n=136$ ) } \\
\hline HLA-DRB $1 * 13$ & 44 & 16.2 & 54 & 10.4 & 0.0228 & 0.3420 & 1.66 & $1.08-2.55$ \\
\hline$H L A-B^{*} 35$ & 53 & 19.5 & 68 & 13.1 & 0.0218 & 0.6758 & 1.67 & $1.08-2.29$ \\
\hline$H L A-B^{*} 44$ & 38 & 14.0 & 46 & 8.9 & 0.0290 & 0.8990 & 1.67 & $1.05-2.64$ \\
\hline $\mathrm{HLA}-\mathrm{B}^{*} 45$ & 0 & - & 12 & 2.3 & 0.0107 & 0.3317 & - & - \\
\hline \multicolumn{9}{|c|}{$A C L$ (group $1 A ; n=118$ ) } \\
\hline$H L A-B^{*} 44$ & 35 & 14.8 & 46 & 8.9 & 0.0160 & 0.4969 & 1.79 & $1.12-2.87$ \\
\hline$H L A-B^{*} 45$ & 0 & - & 12 & 2.3 & 0.0231 & 0.0225 & - & - \\
\hline HLA-DRB $1 * 13$ & 38 & 16.1 & 54 & 10.4 & 0.0152 & 0.2280 & 1.65 & $1.06-2.59$ \\
\hline \multicolumn{9}{|c|}{$\mathrm{ACL}$ (group $2 ; \mathrm{n}=15$ ) } \\
\hline$H L A-B^{*} 27$ & 3 & 10.0 & 8 & 1.5 & 0.0180 & 0.5580 & 7.11 & $1.78-28.33$ \\
\hline \multicolumn{9}{|c|}{$\mathrm{ACL}$ (group $3 \mathrm{~B} ; \mathrm{n}=7$ ) } \\
\hline$H L A-B^{*} 52$ & 3 & 21.4 & 11 & 2.1 & 0.0044 & 0.1360 & 12.61 & $3.08-51.66$ \\
\hline \multicolumn{9}{|c|}{$\mathrm{ACL}$ (group $4 ; \mathrm{n}=22$ ) } \\
\hline HLA-B*49 & 4 & 9.1 & 8 & 1.5 & 0.0101 & 0.3131 & 6.4 & $1.8-22.17$ \\
\hline
\end{tabular}

$\mathrm{n}=$ number of times the allele occurs; $\mathrm{F}=$ Allele frequency; $P$-value $=$ calculated by Fisher's exact test; $P$ c-value $=$ value of $P$ corrected by Bonferroni correction; $\mathrm{OR}=$ odds ratio; $95 \% \mathrm{Cl}=95 \%$ confidence interval.

associated with increased susceptibility: HLA-DRB1 *0407 (OR $=2.14 ; 95 \% \mathrm{CI}=1.34-3.40 ; P=0.001)$ and HLA-DPA $1 * 0401(\mathrm{OR}=10.77 ; 95 \% \mathrm{CI}=1.25-80.73$; $P=0.003)$ [20].

There have been few Brazilian studies on the association between HLA and susceptibility to/protection from cutaneous and mucocutaneous leishmaniasis [16-19], and there is no evidence of any studies in the literature addressing the association between HLA and ACL caused by L. braziliensis in the northwestern region of Paraná, southern Brazil. Studies of the association between HLA and visceral leishmaniasis are underway $[20,21]$; however, this is another form of the disease caused by another Leishmania species and presenting with different clinical manifestations. Thus, we chose not to compare the genetic markers found in these surveys with the results of the present study.

A serological study in Brazil involving L. braziliensis found that allele HLA-DQw3 was associated with risk of infection and HLA-DR2 was associated with protection $(P c=0.004 ; \mathrm{RR}=0.007)$ against mucocutaneous leishmaniasis [18]. The present investigation found no associations with HLA-DR2 in any group.

Preliminary results of the allele frequency of HLA-B*27 $(P=0.0180, P c=0.5580)$ showed a trend toward susceptibility to ACL among patients with the mucocutaneous form. Although the patient samples were too small to draw any definitive conclusions, this finding corroborates that of Petzl-Erler et al. (1991), who analyzed 43 subjects with mucocutaneous leishmaniasis and compared them with 111 controls and initially found the HLA-B27 to be significantly associated $(P=0.029)$, but the significance did not hold after $P$-value correction.

Allele frequency of HLA-DRB1*13 $(P=0.0228, P c=$ 0.3317 ) was conspicuous only in the non-recurrent cutaneous leishmaniasis groups, whereas HLA-B*49 was prominent in the recurrent disease group.

Studies have shown that CD4 + T lymphocytes of the $\mathrm{T}_{\mathrm{h}} 1$ type play a more important role in the immune response to mucosal disease, as there is a major predominance of CD8+ $\mathrm{T}$ lymphocytes in mucocutaneous lesions caused by L. braziliensis [22,23]. The present study has detected a trend toward association with an MHC class I allele (HLA-B*27) in patients with mucocutaneous leishmaniasis, which presents parasite antigens for CD8+ lymphocytes $\mathrm{T}$. It may thus be surmised that a role of CD8+ $\mathrm{T}$ lymphocytes in mucocutaneous leishmaniasis cannot be ruled out.

An immunocytochemical study conducted in the Amazon region found that $\mathrm{CD} 8+\mathrm{T}$ cells occurred at a higher level in all forms of the disease except mucocutaneous leishmaniasis, and included cases of cutaneous lesions caused by L. braziliensis. Its results seem to corroborate the role of CD8 $+\mathrm{T}$ cells in a balanced 
immune response to cutaneous lesions and, probably, in the healing process [23]. In the present study, HLA-B*45 was the only allele associated with a trend toward protection from cutaneous lesions. This corroborates the findings of Silveira et al., 2004, who endorse the role of $\mathrm{CD} 8+\mathrm{T}$ lymphocytes in the healing of cutaneous lesions. Furthermore, HLA-B*45 involvement suggests that the common sequence for the antigen group may anchor parasite peptides and trigger a protecting response. It bears noting that HLA-B*45 is a rare allele in Brazilian populations, with frequencies ranging from $1.0 \%$ to $1.6 \%$ in the state of Paraná [24]. However, its frequency in the control and patient groups was $2.3 \%$ and $0 \%$ respectively. Special attention should be given to this allele in ACL studies.

The frequency of the HLA-A*02-B*44-DRB1*07 and HLA-A*24-B*35-DRB1*01 haplotypes may be involved in susceptibility to cutaneous leishmaniasis. There is evidence in the literature of the involvement of these haplotypes with ACL. Studies have shown occurrence of the HLA-A*02-B*44-DRB1*07 haplotype in populations in the Brazilian states of Maranhão and even in the north of Paraná [25,26].

Research on the epidemiological profiles of ACL shows that infected patients are predominantly male [7,27]. Although the present study did find a male predominance in the infected group, no significant difference between the sexes was found in the control group. Even though males and females are exposed to the same environmental risk factors necessary for ACL acquisition, males are infected with the parasite much more often. The infrequency of ACL in females may be due to their use of repellents to protect themselves during the day and to their relatively infrequent exposure to risk factors, such as being near rivers and in the woods, as compared with men. Furthermore, the sample collection period for the control group may have influenced the number of women in the control group, as women were more likely than men to be home in the morning and afternoon.

In the present study, analysis of MHC class I (HLA-A and HLA-B) and class II (HLA-DRB1) genetic markers failed to show any association with susceptibility or protection to ACL after $P$-value correction. However, all results which initially had significant $P$-values should be carefully observed in further studies, and future studies be conducted with other markers. It bears stressing that, despite the very small sample size of some groups, our findings demonstrate that associations may, in fact, exist between HLA alleles and ACL, and that studies with larger sample sizes are warranted.

\section{Conclusions}

Since the Brazilian population is highly miscegenated, results from studies undertaken in a single region should not be generalized to the whole country. Populations of several other regions should be researched and their allele diversity analyzed so that new associations can be identified or those already known corroborated. Such knowledge shall contribute towards future prophylactic and therapeutic interventions in Brazilian groups at higher risk of developing ACL.

\section{Abbreviations \\ HLA: Human Leukocyte Antigen; MHC: Major Histocompatibility Complex; PCR-SSO: Polymerase chain reaction with sequence-specific oligonucleotides; ACL: American cutaneous leishmaniasis; L. braziliensis: Leishmania (Viannia) braziliensis; F: Allele frequency; HW: Hardy -Weinberg equilibrium.}

\section{Competing interest}

The authors have no conflicts of interest to declare.

\section{Authors' contributions}

RSRC. sample collection; HLA typing; writing of the manuscript. RAD. sample collection; HLA typing. SMCG. - sample collection. JWV. statistical analyses. LMVC. laboratory tests for ACL; writing and revision of the manuscript. BSD. HLA typing; writing and revision of the manuscript STGV. laboratory tests for $\mathrm{ACL}$; writing and revision of the manuscript. All authors have read and approved the submitted version of the manuscript.

\section{Author details}

${ }^{1}$ Postgraduate Program in Health Science, Universidade Estadual de Maringá (UEM), Av. Colombo, 5.790 - Jd. Universitário, Maringá, Paraná 87020-900, Brazil. '2Department of Biostatistics, Universidade Estadual de Maringá (UEM), Av. Colombo, 5.790 - Jd. Universitário, Maringá, Paraná 87020-900, Brazil. ${ }^{3}$ Leishmaniases Laboratory, Department of Clinical Analyses and Biomedicine, Universidade Estadual de Maringá (UEM), Av. Colombo, 5.790 - Jd. Universitário, Maringá, Paraná 87020-900, Brazil. ${ }^{4}$ Immunogenetics Laboratory, Department of Basic Health Sciences, Universidade Estadual de Maringá (UEM), Av. Colombo, 5.790 - Jd. Universitário, Maringá, Paraná 87020-900, Brazil.

Received: 17 August 2012 Accepted: 18 April 2013 Published: 2 May 2013

\section{References}

1. Rey L: Leishmania e leishmaníases: Os parasitos. Parasitologia. Rio de Janeiro: Guanabara Koogan; 2001:214-26.

2. Condino MLF, Galati EAB, Holcman MM, Salum MRB, da Silva DC, Novaes Júnior RA: American cutaneous leishmaniasis on the northern coastline of the State of São Paulo, 1993 to 2005. Rev Soc Bras Med Trop 2008, 41:635-41.

3. Brasil, Ministério da Saúde, Secretaria de Vigilância em Saúde: Manual de vigilância de leishmaniose tegumentar americana. Série A. Normas e Manuais Técnicos. 2nd edition. Brasília: Editora do Ministério da Saúde; 2007. Availeble at: http://www.tedebc.ufma.br/tde_busca/processaPesquisa.php? nrPagina=9. Accessed September 17, 2010.

4. Brasil, Ministério da Saúde, Secretaria de Vigilância em Saúde: Casos de leishmaniose tegumentar americana. Brasil, Grandes Regiões e Unidades Federadas, 1990 a 2008. Brasília: Ministério da Saúde; 2010. Availeble at: http://portal.saude. gov.br/portal/arquivos/pdf/casos_conf_ta.pdf. Accessed September 8, 2010.

5. Lima AP, Minelli L, Teodoro U, Comunello É: Tegumentary leishmaniasis distribution by satellite remote sensing imagery, in Paraná State, Brazil. An Bras Dermatol 2002, 77:681-92.

6. Lonardoni MVC, Teodoro U, Arraes SMAA, Silveira TGV, Bertolini DA, Ishikawa EAY, et al: Leishmaniasis in dogs in Southern Brazil. Rev Saude Publica 1993, 27:378-9.

7. Silveira TGV, Arraes SMAA, Bertolini DA, Teodoro U, Lonardoni MVC, Roberto ACBS, et al: Observations on laboratory diagnosis and cutaneous leishmaniasis epidemiology in the State of Paraná, South of Brazil. Rev Soc Bras Med Trop 1999, 32:413-23.

8. Netto EM, Marsden PD, Llanos-Cuentas EA, Costa JM, Cuba CC, Barreto AC, et al: Long-term follow-up of patients with Leishmania (Viannia) braziliensis infection and treated with Glucantime. Trans $R$ Soc Trop Med Hyg 1990, 84:367-70. 
9. Passos VM, Barreto SM, Romanha AJ, Krettli AU, Volpini AC, Lima e Costa MF: American cutaneous leishmaniasis: use of a skin test as a predictor of relapse after treatment. Bull World Health Organ 2000, 78:968-74.

10. Saravia NG, Weigle K, Segura I, Giannini SH, Pacheco R, Labrada LA, et al: Recurrent lesions in human Leishmania braziliensis infectionreactivation or reinfection? Lancet 1990, 336:398-402.

11. Marsden PD: Mucosal leishmaniasis ("espundia" Escomel, 1911). Trans $R$ Soc Trop Med Hyg 1986, 80:859-76.

12. Cabello PH, Lima AM, Azevedo ES, Krieger H: Familial aggregation of Leishmania chagasi infection in northeastern Brazil. AmJTrop Med Hyg 1995, 52:364-5.

13. Rogers ME, Ilg T, Nikolaev AV, Ferguson MA, Bates PA: Transmission of cutaneous leishmaniasis by sand flies is enhanced by regurgitation of fPPG. Nature 2004, 430:463-7.

14. Handman E, Elso C, Foote S: Genes and susceptibility to leishmaniasis. Adv Parasitol 2005, 59:1-75.

15. Sakthianandeswaren A, Foote SJ, Handman E: The role of host genetics in leishmaniasis. Trends Parasitol 2009, 25:383-91.

16. Barbier D, Demenais F, Lefait JF, David B, Blanc M, Hors J, et al: Susceptibility to human cutaneous leishmaniasis and HLA, Gm, Km markers. Tissue Antigens 1987, 30:63-7.

17. Lara ML, Layrisse Z, Scorza JV, Garcia E, Stoikow Z, Granados J, et al: Immunogenetics of human American cutaneous leishmaniasis. Study of HLA haplotypes in 24 families from Venezuela. Hum Immunol 1991, 30:129-35.

18. Petzl-Erler ML, Belich MP, Queiroz-Telles F: Association of mucosal leishmaniasis with HLA. Hum Immunol 1991, 32:254-60.

19. Olivo-Diaz A, Debaz H, Alaez C, Islas VJ, Perez-Perez H, Hobart O, et al: Role of HLA class II alleles in susceptibility to and protection from localized cutaneous leishmaniasis. Hum Immunol 2004, 65:255-61.

20. Donaghy L, Gros F, Amiot L, Mary C, Maillard A, Guiguen C, Gangneux JP: Elevated levels of soluble non-classical major histocompatibility class I molecule human leucocyte antigen (HLA)-G in the blood of HIV-infected patients with or without visceral leishmaniasis. Clin Exp Immunol 2007, 147(2):236-40

21. Peacock CS, Sanjeevi CB, Shaw MA, Collins A, Campbell RD, March R, Silveira F, Costa J, Coste CH, Nascimento MD, Siddiqui R, Shaw JJ, Blackwell JM: Genetic analysis of multicase families of visceral leishmaniasis in northeastern Brazil: no major role for class II or class III regions of HLA. Genes Immun 2002, 3(6):350-8.

22. Silveira FT, Lainson R, Corbett CE: Clinical and immunopathological spectrum of American cutaneous leishmaniasis with special reference to the disease in Amazonian Brazil: a review. Mem Inst Oswaldo Cruz 2004, 99:239-51.

23. Silveira FT, Lainson R, De Castro Gomes CM, Laurenti MD, Corbett CE: Immunopathogenic competences of Leishmania (V.) braziliensis and L. (L.) amazonensis in American cutaneous leishmaniasis. Parasite Immunol 2009, 31:423-31.

24. Allele Frequencies. ; 2010. Availeble at: http://www.allelefrequencies.net/ Accessed September 17, 2010.

25. Ferreira FL, Mesquita ERRBPL: Polimorfismo Genético do Sistema HLA em uma amostra de Doadores Voluntários de Medula Óssea do Maranhão. Dissertação de Mestrado do Centro de Ciências Biológicas e da Saúde. São Luís:

Universidade Federal do Maranhão; 2007. Available at: http://www.tedebc. ufma.br//tde_busca/arquivo.php?codArquivo=287 Accessed July 17, 2012.

26. Gonçalves MSB, Jarduli LR, Jorge AJ, Camargo RBOG, Carneiro FP, Gelinski JR, Silva JC, Silva RAF, Lavado EL: Freqüência alélica e haplotípica HLA-A, B e DRB1 em doadores voluntários de medula óssea na população norte-paranaense. Temas livres/Abstracts - Histocompatibilidade. Rev Bras Hematol Hemoter 2010, 32(3):13-25.

27. Silveira TGV, Teodoro U, Lonardoni MVC, Guilherme ALF, de Toledo MJO, Ramos $M$, et al: Epidemiologic aspects of Cutaneous Leishmaniasis in an endemic area of the state of Paraná, Brazil. Cad Saude Publica 1996, 12:141-7.

doi:10.1186/1471-2334-13-198

Cite this article as: Ribas-Silva et al:: Association between HLA genes and American cutaneous leishmaniasis in endemic regions of Southern Brazil. BMC Infectious Diseases 2013 13:198.

\section{Submit your next manuscript to BioMed Central and take full advantage of:}

- Convenient online submission

- Thorough peer review

- No space constraints or color figure charges

- Immediate publication on acceptance

- Inclusion in PubMed, CAS, Scopus and Google Scholar

- Research which is freely available for redistribution

Submit your manuscript at www.biomedcentral.com/submit
C) BioMed Central 